Asian Journal of Information Technology 10 (7): 281-285, 2011

ISSN: $1682-3915$

(C) Medwell Journals, 2011

\title{
Spatial Auditory Based Interaction Model for Driver Assistance System
}

\author{
Dalbir Singh \\ Faculty of Information Science and Technology, \\ Universiti Kebangsaan Malaysia, Bangi, Selangor, Malaysia
}

\begin{abstract}
The expansion of Malaysia's automobile industry has witnessed the emergence of Driver Assistance System (DAS) that enables the interaction between the driver and the vehicle. Over the past few years, DAS has become a necessity and is commonly found on conventional vehicles instead of as luxury equipments on high-end vehicles. The rapid development of DAS provides drivers with radically enhanced information and functionality. The nature of the current DAS requires a complex human-machine interaction which is distracting and may increase the risk of road accidents. The interaction between the driver and DAS should aid the driving process without interfering with safety and ease of vehicle operation. Speech based interaction mechanisms employed are not sufficiently robust to deal with the noise present in the interior environment of the vehicle. Simultaneous speech based interaction from components of DAS would annoy and increase the driver's cognitive burden. Therefore, suitable hybrid spatial auditory interaction principles for DAS are developed on principles that utilize concurrent auditory data transmission and its location source of origin in the in vehicle environment. The proposed model exploits four auditory sources in the vehicle's interior environment. These interfaces are investigated in driving simulator experiments in order to test their durability and robustness. This will ensure the driving-related information from the DAS is delivered to the driver without affecting the overall driving process.
\end{abstract}

$\underline{\text { Key words: Driver assistance system, speech, cognitive workload, spatial auditory, interaction, vehicle }}$

\section{INTRODUCTION}

Currently, most of the driver assistance systems are derived from a visual-based interaction channel which displays complex information at a high rate (Kim et al., 2008). As this requires a high visual processing capability, the driver's attention to the driving task is considerably reduced. Hence, there is a tendency for information to be misread as the driver's attention ceases and is not focused at the right place due to visual information overload. Moreover, these driver assistance systems increase the driver's reaction time to detect an event or incident and reduce the view of the road whilst driving. The in vehicle environment becomes informationintensive where the visual channel is overloaded hence, unnecessarily jeopardizing the driver's and the passenger's safety (Michalke et al., 2010). Auditory (speech and non-speech) based interaction for driver assistance system is known to reduce the reaction time towards event detection and poses lesser danger to the driver (Bellotti et al., 2002). Improper application of synthetic speech based interaction for driver assistance system can have hazardous implications (Nirschl, 2007). This is because synthetic speech-based interaction poses high cognitive load on the driver's short term memory (auditory-speech processing capabilities). The use of synthetic speech may also be annoying for some driver and loses its prosodic information.

Furthermore, the driver is loaded with other incoming speech-based interactions from the different components of DAS while driving. The driver assistance system sometimes requires the driver to perform synchronous interactions with the vehicle's control mechanisms (Bellotti et al., 2002). The cognitive ability of the driver is reduced especially in a demanding driving situation (Bellotti et al., 2002; Nirschl, 2007).

According to a survey conducted among Malaysian drivers, the drivers are burdened cognitively from interactions from various components of the DAS simultaneously while driving. Besides that ADAS is considered as distractions and would be turned off while driving. This does not enable the driver to harness the full potential of DAS. Since, the driver's distraction becomes a significant problem, the human-machine interface needs to be designed as efficiently as possible. These driver assistance systems must function without decreasing safety as well as ease the overall driving process (Nirschl, 2007). 


\section{MATERIALS AND METHODS}

\section{Hybrid spatial auditory based DAS interaction research} issues: In this study, auditory based interaction model is proposed to complement the existing visual based interface. It is an alternative to synthetic non-speech based interaction for driver assistance system (Gerhing and Morgan, 1990). The proposed model will be tested in the driving simulator experiments. These research issues must be borne in mind when creating an auditory interaction mechanisms for DAS.

Spatial auditory environment: In this study, researchers have examined some previous research efforts in this area and it is proven that humans are able to identify the location of the sound's source (Marentakis and Brewster, 2005; Gerhing and Morgan, 1990). This concept has not been tested for in-vehicle environment and no auditory icon design principles have been tested for such purpose. The main intention is to harness the benefits of the spatial audio environment that enable us to deliver the needed driving-related information without burdening the driver cognitively. According to previous research, one of the problems is to deliver a high volume of information through a single audio channel (Ekholm, 2002; Tonnis and Klinker, 2006). Spatial audio environment offer multiple audio channel. Another issue arises, how do we arrange and categorize such information so that it is presentable to the user while driving? (Riecke et al., 2009). In this study, we have proposed five interactions and presentation architectures to tackle this issue. These are the basic principles of the proposed spatial auditory model that utilize a spatial auditory interaction that enables concurrent auditory information to be transmitted to the driver while driving. The creation of the auditory information is based on three categories of the DAS's components: Safety, Navigation and Entertainment/ Communication.

These categories are derived from previous research that investigates the information presentation issues for DAS (Marcus, 2004). Besides that these categories present the major components of modern DAS and widely used approach (Lerner et al., 2008). The auditory information is dynamically transmitted (concurrently) from four spatial auditory sources. The cases (1-5) demonstrate five different architectures for transmitting auditory information in a spatial auditory environment as shown in Fig. 1-5. The experiments for case 1-5 would determine the best spatial auditory model for concurrent information transmission in-terms of safety and usability.

Case 1: In case 1, safely is tagged as the highest priority followed by navigation and lastly entertainment/

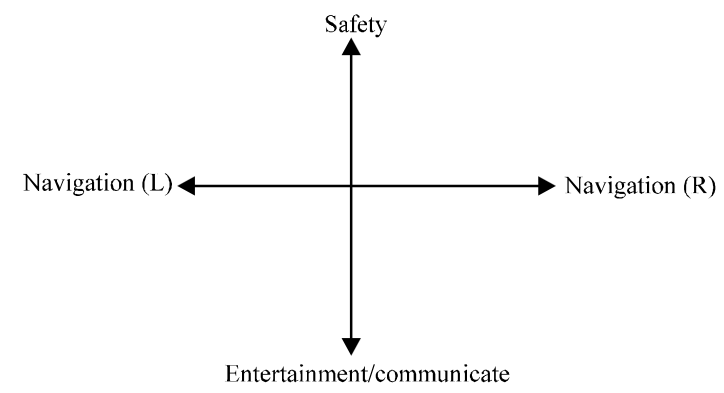

Fig. 1: Spatial audio architecture for case 1

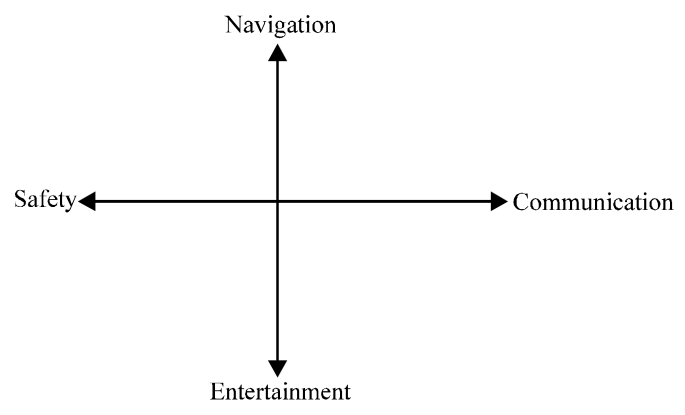

Fig. 2: Spatial audio architecture for case 2

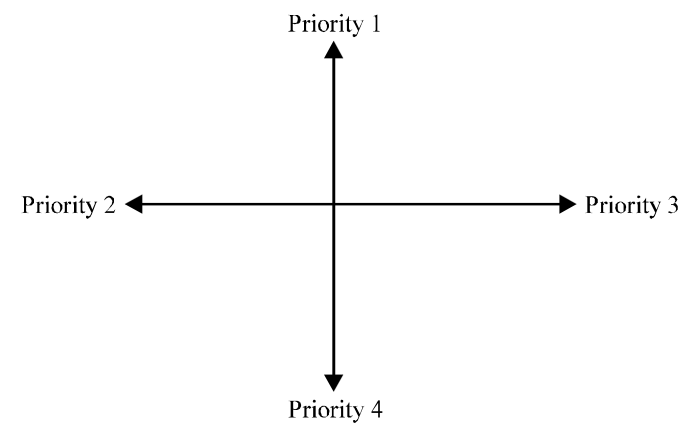

Fig. 3: Spatial audio architecture for case 3

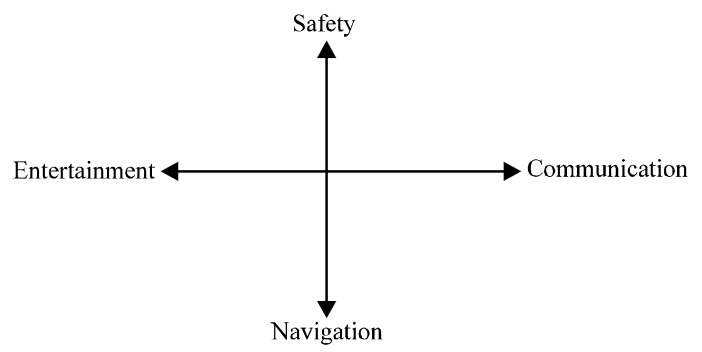

Fig. 4: Spatial audio architecture for case 4

communication. The navigational direction for the turning purpose utilizes left/right audio source and both for the start/stop signal. Overall, a 4 channel spatial audio is employed that emitted driving related information from 4 sound sources (Fig. 1). 
Asian J. Inform. Technol., 10 (7): 281-285, 2011

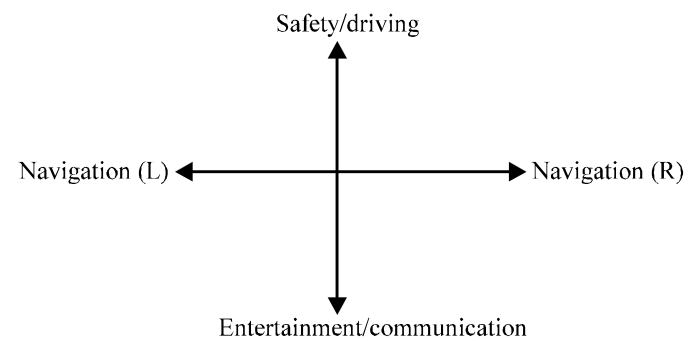

Fig. 5: Spatial audio architecture for case 5

Case 2: In case 2, navigational information is given the highest importance but in case of hazardous situation. Safely is given the most priority. This shows the priority ranking is dynamic but the audio output source's location remains the same. In other words what changes is the frequency and amplitude (Fig. 2).

Case 3: In case 3, each audio source channel is rated according to the priority ranking based on the diagram on the left. The driving information's categories changes their location based on their priority. The frequency and amplitude is also based on priority ranking (Fig. 3).

Case 4: In sub-case 4, the main objective is to test the effectiveness of the front/back audio source in a 4 auditory channel spatial environment. It is different from the previous experiment that only test the front/back without considering the concurrent effect of the left and right auditory (Fig. 4).

Case 5: In sub-case 5, the main objective is to test the priority mechanism with a single auditory channel that emphasizes the front/back auditory channel. Concurrently the left and right auditory channel provides the navigational cues. For the front/back auditory channel, the driving information is emitted at different frequences and amplitudes (Fig. 5).

Spatial auditory environment: The driving simulation experiments involve the use of computer based vehicle controls (steering/pedals/panels) and a driving simulator to create a real-time driving environment. This will ensure the validity and integrity of the results produced by these driving experiments (Cha et al., 2008). The subject is supposed to drive in a computer-based driving simulator through a series of roads that include a few junctions/traffic lights in a medium traffic condition. Before the experiment, the subject is given $15 \mathrm{~min}$ to test drive freely in the driving simulator. The subject does not know the route of the driving course. The subjects would not drive on the same route more than once (different routes and driving conditions). Therefore, these

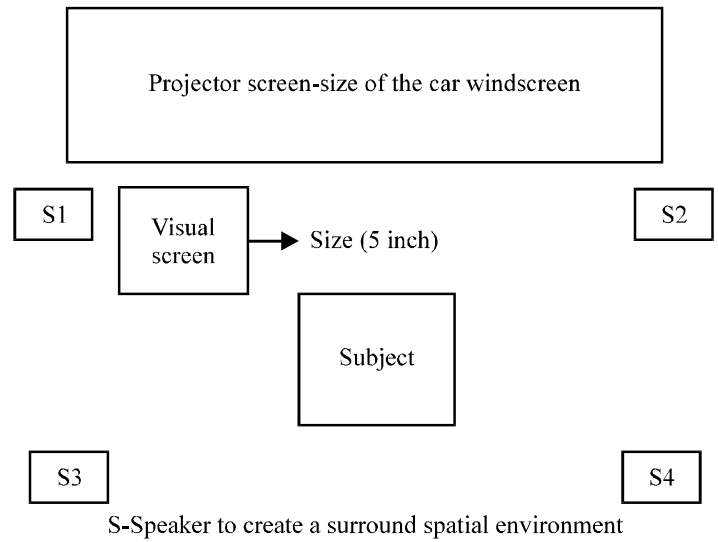

Fig. 6: Driving simulation experiment layout

experiments are not repeated to ensure maximum dependability of the driver on the DAS. During the driving experiment, infotainment (radio), safety (accident avoidance) and road direction information are delivered to the subjects. This will simulate a real-time spatial auditory environment in a driving situation as it would provide the same cognitive load with the real time driving situation as shown in Fig. 6.

The projector screen displays the road scene and the internal view of the vehicle's panel and steering. Indirectly, the projector screen acts as the windscreen of the vehicle. The 5 inch visual screen (monitor) displays the DAS. The subjects are supposed to drive in the driving simulator based on the direction from the DAS (auditory sources surrounding the subject (S1-S4)). The subjects are tested in the driving simulator according to three parameters: Correct turning (total of 10 correct turnings), respond time towards prompts (5 prompts) and comprehension test (15 questions). The comprehension test is conducted based on the information emitted from the entertainment/communication channel.

\section{RESULTS AND DISCUSSION}

Case 1 result: In case 1 , the subjects were able to differentiate the left and right auditory channel as the navigational guide. The auditory sound location provides an extra guide besides the use of different earcons. The performance decreases when the subjects are required to listen to communication messages (speech) and react to the safety notifications. The subjects are cognitively burdened and are not able to react accordingly to the notification represented to them.

Case 2 result: In case 2, the subjects are able to focus on certain notifications based on the priority mechanism 


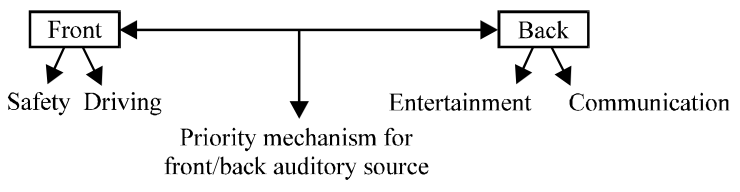

Fig. 7: Front/back auditory channel information management

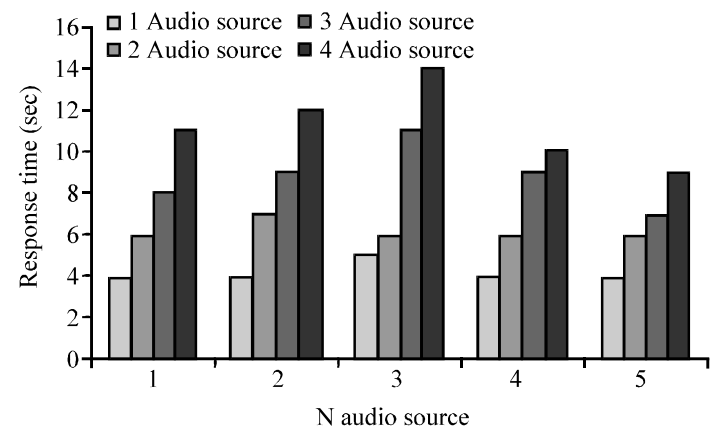

Fig. 8: Response time for case $1-5$ with an increasing auditory source

used in this case study. The disadvantage of the navigational sound source in front is that it eliminates the auditory clue. Furthermore, auditory notification also burdens the subjects cognitively and increases the reaction time. Therefore, three or more notifications cannot be understood by the subjects even if the notification mechanism is used.

Case 3 result: In case 3 , the subjects got lost while the messages are emitted from each auditory source as the priority changes. The subjects complain they could not focus if the priority changes and it affects the overall performances.

Case 4 result: In case 4 , the subjects are able to focus more on the changing priority between the front/back auditory channels compared to the left and right auditory source. It shows us that changing the priority between two sources while concurrently playing messages or notification from other sources is more desirable.

Case 5 result: Case 5 shows us an improved performance compared to case 4 . Instead of changing priority between two auditory sources only, researchers have tested changing sub-priority for each auditory channel (front/back). This creates an extended priority mechanism where more information can be represented to the driver in a more orderly manner as shown in Fig. 7.

The left and right auditory source provides the additional navigational cues as proven in case 1 . The subjects have a better response time even when three or more information is simultaneously represented. The presentation mechanism plays an important role in coordinating the front/back notification transmission.

The response time increases as more spatial auditory location transmit information as shown in Fig. 8. The response time got worse when a maximum of four auditory sources transmit information simultaneously. The response time improves for cases 4 and 5 even when the amount of information increases relatively. In both of these cases, the front/back auditory channel has been put into test and it shows that the subjects are able to understand notification/information (speech) that changes its amplitude according to the dynamic priority. Previous cases show us that left and right auditory sources provide additional navigational cues besides having different auditory icons. This also proves that changing the amplitude according to the front/back auditory presentation model provides a suitable mechanism for simultaneous information transmission (speech and non-speech)

This proves the proposed spatial auditory interaction design principles are suitable with the presentation mechanism for in vehicle environment. Therefore, the hypothesis is proven true. From the analysis and discussion based on these experiments, a new DAS information presentation model that transmitted auditory information for an in-vehicle environment is obtained.

\section{CONCLUSION}

In this study a holistic approach for information mapping (visual into non-speech) and auditory presentation model is proposed. The whole DAS was considered from entertainment devices to safety-related systems.

The issues concerning the driver's cognitive load and information presentation were considered. The proposed auditory interaction model that consists of a central interaction mechanism that controls the interaction from three sub-systems of the DAS based on auditory source location's clues. The novel contribution of this thesis can be used as a guideline for future application of auditory interface for DAS.

Besides that one surprising discovery has opened the frontier for future research work based on this research project. The subject shows the pattern of preference based on their racial and educational background. Further research work would be carried out to investigate the cross-cultural adaptation factors for the usage of DAS among Malaysian drivers. 


\section{REFERENCES}

Bellotti, F., R. Berta, A. De Gloria and M. Margarone, 2002. Using 3D sound to improve the effectiveness of the advanced driver assistance systems Pers. Ubiquitous Comput., 6: 155-163.

Cha, M., J. Yang and S. Han, 2008. hybrid driving simulator with dynamics-driven motion and datadriven motion. Simulation, 84: 359-371.

Ekholm, A., 2002. The design of in-car communication and information applications. Pers. Ubiquitous Comput., 6: $153-154$.

Gerhing, B. and D. Morgan, 1990. Applications of binaural sound in the cockpit. Speech Technol., 5: 46-50.

Kim, S.Y., J.K. Kang, S.Y. Oh, Y.W. Ryu, K. Kim, S.C. Park and J. Kim, 2008. An intelligent and integrated driver assistance system for increased safety and convenience based on all-around sensing. J. Intell. Robot. Syst., 51: 261-287.

Lerner, N., J. Singer and R. Huey, 2008. Driver Strategies for Engaging in Distracting Tasks Using in Vehicle Technologies. Technical Report Documentation Page, National Highway Traffic Safety Administration, U.S. Department of Transportation, pp: $16-60$.
Marcus, A., 2004. The next revolution: Vehicle user interfaces. Interactions, 77: 40-47.

Marentakis, G. and S.A. Brewster, 2005. A comparison of feedback cues for enhancing pointing efficiency in interaction with spatial audio displays. Proceedings of the 7th International Conference on Human Computer Interaction with Mobile Devices and Services, Sept., 19-22, Saizburg, Austria, pp: 55-62.

Michalke, T., J. Fritsch and C. Goerick, 2010. A biologically-inspired vision architecture for resourceconstrained intelligent vehicles. Comput. Vision Image Understanding, 11: 548-563.

Nirschl, G., 2007. Human-centered development of advanced driver assistance systems. Proc. Conf. Hum. Interface, 2: 1088-1097.

Riecke, B.E., A. Valjamae and J. Schulte-Pelkum, 2009. Moving sounds enhance the visually-induced selfmotion illusion (circular vection) in virtual reality. ACM Trans. Applied Percept., 6: 1-25.

Tonnis, M. and G. Klinker, 2006. Effective control of a car driver's attention for visual and acoustic guidance towards the direction of imminent dangers. Proceedings of the 5th IEEE and ACM International Symposium on Mixed and Augmented Reality, Oct. 22-25, Santa Barbard, CA., pp: 13-22. 